\title{
Microeconomic Evolution Model with Technology Diffusion
}

\author{
K. $\mathrm{CICHY}^{*}$ \\ Chair of Mathematical Economics, Poznań University of Economics, al. Niepodleglosci 10, 60-967 Poznań, Poland \\ Faculty of Physics, Adam Mickiewicz University, ul. Umultowska 85, 61-614 Poznań, Poland
}

\begin{abstract}
The model discussed in this paper is a modification of the model of macroeconomic evolution in stable regionally dependent fields, developed by Ausloos, Clippe and Pekalski in 2004. Like in the original model, firms exist on a square lattice and can move, merge, adapt and create spin-offs. However, in the new model the firms are described by a scalar parameter identified with their level of technology and by their market share. The probability of survival of a firm depends on the relation between the firm's technology level and the level of the technological frontier. The model incorporates two mechanisms of technology diffusion - inner (resulting from the cooperation between firms and the creation of spin-offs) and outer (interaction with the technological frontier). In this way, we obtain a model of technological progress with technology diffusion. We investigate the properties of this model and perform empirical analysis for a group of OECD countries.
\end{abstract}

PACS: 89.65.Gh, 88.05.Lg

\section{Introduction}

One of the most important sources of economic growth is believed to be knowledge. In economic growth theory, knowledge is usually embodied in technology and/or in human capital. However, one of the most important questions is how to effectively model technology and the phenomenon of technological progress, i.e. the improvements in technology.

Economic growth theory dates back to the beginning and the middle of the 20th century, when the ideas of Adam Smith [1] and other economists were formalized in the language of mathematics. The pioneering works of economic growth theory include the works of Ramsey [2], Solow [3] and Swan [4], Cass [5] and Koopmans [6]. The key mechanism in the models of Ramsey-Cass-Koopmans and Solow-Swan is the accumulation of physical capital, which, however, can not explain economic growth, if we understand it as the growth of production per person. To achieve growth, one needs an extra production factor - usually termed technology or human capital.

In this paper, we will concentrate on technology and technological progress. The simplest way to obtain economic growth in the Solow-Swan or the Ramsey-CassKoopmans model is to add technology, understood as an additional variable that grows at a constant rate, without any attempts to understand the mechanism behind this growth. Such extension of the model shows that even if the accumulation of physical capital is not enough to explain economic growth, growth may result from the increasing efficiency of combining capital and labour.

The branch of economic growth theory that follows this line of reasoning and looks for the source of economic growth in this direction, is called endogenous growth theory. The main aim of endogenous growth theory is to find

\footnotetext{
* e-mail: krzysztof.cichy@ue.poznan.pl, kcichy@amu.edu.pl
}

plausible mechanisms of technological progress. In combination with physical and human capital accumulation and population growth, it should explain why some countries are poor and some are rich, and why production and wealth grows faster in some countries and slower in some other countries.

The pioneering and widely regarded as the most important models of endogenous growth theory were formulated around 1990 by Romer [7] (model of technological progress) and Lucas [8] (model of human capital). Since then, several models with different assumptions and different mechanisms of technological progress and/or human capital accumulation have been proposed. It is not the aim of this paper to review these models - for such review we refer to e.g. [9].

In addition to the mainstream economics approach to technological progress, this issue, and in general the subject of economic growth, becomes a topic of interest also to physicists. Physicists are interested in economics for a few reasons, the foremost being that they realized that economic systems are very complex systems which can be analyzed with the tools of statistical physics. Among the many interesting works in Econophysics it is worth to point to collections of papers edited by Chakrabarti et al. [10] and Gallegati et al. [11]. However, the emphasis of Econophysics is usually not on economic growth.

Among the few Econophysics papers related to technological progress and/or economic growth it is worth to mention e.g. the articles by Ausloos et al. [12], Delli Gatti et al. [13], Llas et al. [14] and Szydlowski with Krawiec [15]. Usually, such papers do not explicitly mention economic growth, but it is clear from their formulation that they can be interpreted as models of technological progress. Sometimes, however, a reinterpretation of a model may be needed.

It is the aim of this paper to provide an extension to the model of Ausloos et al. [12] and to reinterpret some of its components and notions in such a way that it explicitly 
concerns technological levels and technological advancement of firms. We will also propose a method of performing empirical research based on this class of models. The described model was first discussed in the Ph.D. thesis of the author of this paper [9].

The paper is organized as follows. In Section 2 we give the setup of the model. Section 3 discussed the properties of the model. In Section 4 we present the result of empirical analysis for the group of OECD countries. Section 5 concludes.

\section{Setup of the model}

We consider a simple economy with $N(t)$ firms $(t$ denotes discrete time step). The characteristics of each firm includes its technology level, denoted by $A_{i}(t)$ and its relative market share $w_{i}(t)$ (such that $\forall t \sum_{j} w_{j}(t)=1$ ), where the subscript $i$ denotes firm $i$.

The firms occupy the sites of a square lattice with $L_{x} \times$ $L_{y}$ sites. Each site can be occupied by one firm or can be unoccupied. The initial distribution of firms is described by the concentration parameter $c_{0}=N(0) / L_{x} L_{y}$. An example of such distribution is shown in Fig. 1.

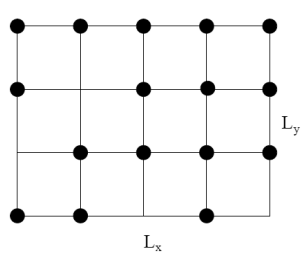

Fig. 1. An example of the initial distribution of firms (16 firms, concentration $c_{0}=80 \%$ ).

We assume that the initial distribution of technology levels is uniform on the interval $(0,1)$ and initially all firms have the same market share $\forall i w_{i}(0)=1 / N(0)$. To describe the average level of technology and take into account the varying market share of firms, we introduce the weighted average level of technology for the considered economy $\langle A(t)\rangle$ (we have $\langle A(0)\rangle \approx 0.5$ ).

The dynamics of firms in the considered economy depends on their technology levels, the technology levels of their competitors and the frontier (world-leading) technology level $F(t)$, which is exogenous with respect to the firms and is given by:

$$
F(t)=e^{\sigma t}
$$

where $\sigma$ is a parameter which measures the world technological progress rate.

The most important assumption about the firms is that the probability of survival of a firm depends on their technology - if a firm's technological level is high enough (what this precisely means will be specified below), this firm is certain to survive, on the other hand the probability of bankruptcy increases in the case of lower levels of technology.
If a firm survives, it takes up activities to increase its technology level and hence its survival probability in following time steps. These activities consist in moving through the lattice to look for a partner for collaboration (merging with other firms or creating spin-offs).

We also assume that there is a lower bound on the number of firms in the system - a decreasing number of competitors makes it easier for the surviving firms to stay on the market and the number of firms never goes below some specified $N_{\text {min }}$.

\subsection{Monte Carlo simulations algorithm}

We now specify the Monte Carlo algorithm for an arbitrary time step $t$. See also comments to the algorithm below at places marked by an asterisk $(*)$.

1. Randomly choose a firm indexed by $i$ from the set of $N(t)$ firms.

2. Calculate its survival probability $(*)$ :

$$
p_{i}= \begin{cases}e^{-s\left(\langle A(t)\rangle F(t)-A_{i}(t)\right)} & \\ & \text { if }\langle A(t)\rangle F(t)>A_{i}(t),\langle A(t)\rangle<1 \\ e^{-s\left(F(t)-A_{i}(t)\right)} & \\ & \text { if } F(t)>A_{i}(t),\langle A(t)\rangle \geq 1 \\ 1 & \\ & \text { if } A_{i}(t) \geq\langle A(t)\rangle F(t),\langle A(t)\rangle<1 \\ 1 & \\ & \text { if } A_{i}(t) \geq F(t),\langle A(t)\rangle \geq 1 .\end{cases}
$$

3. Draw a number $r$ from the uniform distribution $\left(^{*}\right)$. $3 \mathrm{a}$. If $r>p_{i}$, the firm goes bankrupt and the lattice site becomes empty. The market shares of other firms grow proportionally, such that the normalization condition $\sum_{i} w_{i}(t)=1$ holds. We return to point 1 of the algorithm.

3b. If $r \leq p_{i}$, the firm survives and tries to move to a neighbouring lattice site. We draw a number $r_{1}$ and if $r_{1}<0.25$, we check whether the site which is north of the firm's site is empty, if $0.25 \leq r_{1}<0.5$ we check the site which is west etc.

4. If the neighbouring site is empty, the firm moves to this site and checks whether there is some other firm in the nearest neighbourhood of the new site. If such firm is absent, the firm's technology is increased according to the formula $(*)$ :

$$
A_{i}(t) \rightarrow A_{i}(t)+r_{2}\left(F(t)-A_{i}(t)\right)
$$

where $r_{2}$ is a number drawn from the uniform distribution. Then we come back to point 1 of the algorithm.

5. If there is some firm $j$ in the neighbourhood of the firm $i$, then:

5a. with probability $b$ (which is a parameter of the model), the firms merge. The technology of the new firm is $(*)$ :

$$
\begin{array}{cl}
\text { VAR. 1: } & A_{i}(t) \rightarrow 0.5\left(A_{i}(t)+A_{j}(t)+\right. \\
& \left.0.5 r_{3}\left|A_{i}(t)-A_{j}(t)\right|\right), \\
\text { VAR.2: } & A_{i}(t) \rightarrow \max \left\{A_{i}(t), A_{j}(t)\right\},
\end{array}
$$

where $r_{3}$ is a number drawn from the uniform distribution. The firm $j$ disappears from the system and the 
market share of the new firm is equal to the sum of market shares of the merging firms.

5b. With probability $1-b$, the firms $i$ and $j$ create a spin-off. A firm $k$ emerges and it is located in the 8-site neighbourhood of the firm $i$ (north, north-west, west, south-west etc.). The positioning procedure is analogous to the one in point 3 (a number $r_{4}$ is drawn and depending on the outcome a suitable site is chosen). If the appropriate site is not empty, the spin-off does not emerge. The technology of the spin-off equals (there are again two variants, analogous to the case when firms merge):

$$
\begin{array}{cl}
\text { VAR. 1: } & A_{i}(t) \rightarrow 0.5\left(A_{i}(t)+A_{j}(t)+\right. \\
& \left.0.5 r_{5}\left|A_{i}(t)-A_{j}(t)\right|\right) \\
\text { VAR.2: } & A_{i}(t) \rightarrow \max \left\{A_{i}(t), A_{j}(t)\right\}
\end{array}
$$

where $r_{5}$ is a number drawn from the uniform distribution. The market share of the new firm is equal to the sum of market shares of firms $i$ and $j$, multiplied by a parameter $w_{s} \in[0,1](*)$. The market shares of firms $i$ and $j$ decrease accordingly, by $w_{i} w_{s}$ and $w_{j} w_{s}$.

6 . We return to point 1 of the algorithm until $N(t)$ firms have been chosen. Then, a Monte Carlo step is finished, i.e. we set $t \rightarrow t+1\left({ }^{*}\right)$.

\subsection{Comments to the algorithm}

Ad. 2. The system's sensitivity to technological backwardness is expressed by the value of the parameter $s$, i.e. higher values of this parameter mean that the survival probability is lower for firms whose technology level is off the frontier value. If $s=0$, in turn, then the system is insensitive with respect to technological backwardness and firms never go bankrupt. We distinguish two phases, with different influence of the frontier technology:

- phase when the average technology has not yet reached the level $F(0)=1$ - the first and third formula of eq. (2) imply that a firm is certain to survive if their level of technology is equal to at least $\langle A(t)\rangle F(t)$; when it is lower than this value, a non-zero probability of bankruptcy occurs,

- phase when the average technology in the system has reached $F(0)=1$ - the second and fourth formula of eq. (2) imply that a firm is certain to survive if their level of technology is not worse than the frontier technology $F(t)$; when it is lower than $F(t)$, there is a non-zero probability of bankruptcy.

The existence of the first phase is thus an additional advantage of the countries in which the technology is relatively bad and makes possible a faster growth by making sure that the best firms are certain to survive, even if their technology level is still worse than the initial level of the frontier technology.

ad. 3. By uniform distribution we mean the uniform distribution on the interval $(0,1)$, unless otherwise stated.

ad. 4. Eq. (3) expresses a technology diffusion effect,

which we will call outer technology diffusion. Outer technology diffusion means the imitation of the frontier technology. However, usually it is not possible to introduce the frontier technology fully and the level of technology of the firm grows by a fraction $r_{2}$ of the difference of the frontier technology and the current technology of the firm.

ad. 5a. In the first variant, the technology of the new firm is the arithmetic mean of the technology levels of the merging firms plus some synergy effect, which is larger if the difference in technologies is larger. In the second variant, the new firm's level of technology is equal to the technology level of the technologically more advanced firm. Such synergy effects will be called inner technology diffusion.

ad. 5b. The extreme values of the parameter $w_{s}$ correspond to the cases that the spin-off can never emerge $\left(w_{s}=0\right)$ or if the firms $i$ and $j$ disappear when the spinoff emerges $\left(w_{s}=1\right)$.

The economy profits from inner technology diffusion also if a spin-off is created.

ad. 6. The random choice of a firm in point 1 of the algorithm implies that a given firm can be chosen more than once at time $t$ and hence firms which are not chosen at this Monte Carlo step exist.

(a)
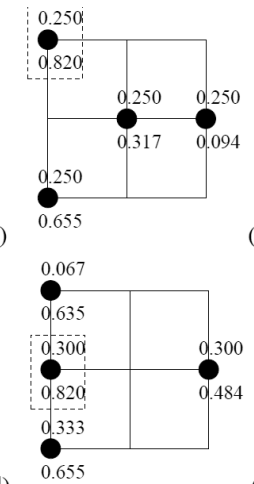

(d)

(e)

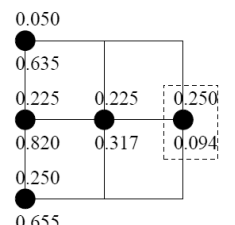

(b)
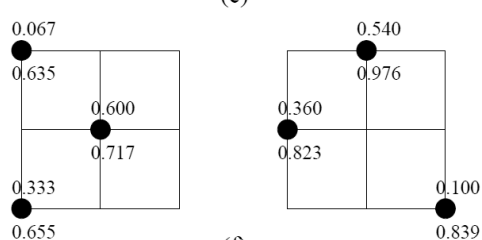

(f)

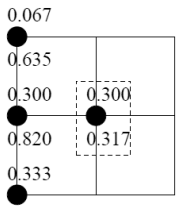

Fig. 2. An example economic evolution of a $3 \times 3$ system. Explanation: parameter values: $\sigma=0.01, s=1$, $b=0.1, w_{s}=0.1$. The upper number denotes the weight, the lower number the technology level.

\subsection{Example}

We will now shortly discuss an example economic evolution according to the above specified algorithm, for a simple $3 \times 3$ system with $N(0)=4$. Fig. $2($ a) shows that the firm $(1,1)$ (we number the sites like entries of a matrix, starting from the upper left corner) was chosen. The probability of survival of this firm equals 1 , since the level of technology of this firm exceeds $\langle A(0)\rangle=0.472$. The firm moves to site $(2,1)\left(r_{1}=0.726\right)$ and creates a spin-off with firm $(2,2)$. The spin-off emerges at site $(1,1)$. The market share of the spin-off is 0.05 and the level of technology is larger than the arithmetic mean of the parent-firms - thus the average technology in the 
system grows to 0.475 (Fig. 2(b)). Hence, we observe $i n$ ner technology diffusion. Next, the firm $(2,3)$ is chosen. Its probability of survival is 0.683 . We draw $r=0.701$ and the firm $(2,3)$ goes bankrupt. The (weighted) average level of technology of the remaining firms is 0.602 . In Fig. 2(c), the firm $(2,2)$ is chosen. Its survival probability equals 0.752 . We obtain $r=0.123$ (the firm survives) and moves to site $(2,3)\left(r_{1}=0.916\right)$. The lack of neighbours means that we observe the outer technology diffusion and the level of technology of this firm goes up from 0.317 to 0.484 ; the average technology $\langle A(0)\rangle$ rises to 0.652 . In Fig. 2(d) the firm $(2,1)$ is chosen. The survival probability is 1 and the firm moves to site $(2,2)$ and merges with firm $(2,3)$. The new firm's technology is the arithmetic mean of the parent-firms plus some synergy effect (inner technology diffusion). We obtain the situation depicted in Fig. 2(e). The zeroth Monte Carlo step is finished (the algorithm involved $N(0)=4$ firms) and the average level of technology is now 0.691. Fig. 2(f) shows an example of the situation after 10 Monte Carlo steps. At this time, there are 3 firms in the system and the average technology level equals $\langle A(10)\rangle=0.907$. However, the technology frontier moved to $F(10)=1.105$, so relatively to the technological frontier, the average level of technology is now $\langle A(10)\rangle / F(10)=0.821$.

\section{The properties of the model}

We now show the simulation results for a $10 \times 10$ system, with an initial concentration of firms equal to 0.8 and the following parameters: $\sigma=0.01, s=1, b=0.01$, $N_{\text {min }}=10, w_{s}=0.1$. This will allow us to draw some general conclusions about the properties of the model and the types of dynamics that it describes.

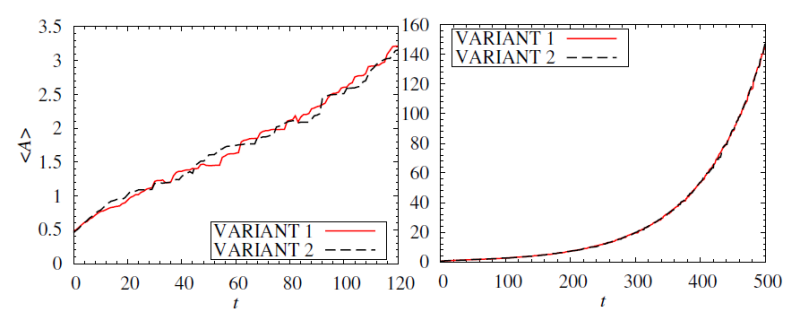

Fig. 3. Average technology dynamics in the mid- and long-term.

Figs. 3-5 show the dynamics of the system (the average technology $\langle A\rangle$, the average technology relatively to the frontier technology $\langle A\rangle / F$ and the number of firms $N$ ) in two time horizons, which we call the mid- (left panels) and long-term (right panels). We can distinguish three phases in the dynamics of the considered economy.

1. Initial phase - fast decrease in the number of firms (the firms with the worst technology disappear), approximately linear growth of average technology $\langle A\rangle$, fast growth in average relative technology $\langle A\rangle / F$. This phase lasts for 20-30 Monte Carlo steps (for chosen parameter values).
2. Non-stable equilibrium phase - the number of firms fluctuates around ca. $50 \%$ of the initial number (ca. $50 \%$ of the initial number of firms have $A(0)<\langle A(0)\rangle$; in the second variant the number of firms is a little larger, since when the firms merge, the new firm has better technology than in variant 1 ; also, the transitions between phases are much clearer in variant 1 than in variant 2 ), approximately linear growth in average technology (at a rate similar to phase 1) and a much slower growth in average relative technology $\langle A\rangle / F$ (since the average technology is already close to its frontier value). This phase lasts for around 100 Monte Carlo steps.

3. Stable equilibrium phase - at some time step there is a sudden drop in the number of firms. In the second phase, the number of firms was $40 \pm 10$ for ca. 100 steps; here it drops to 10 in only ca. 10 steps (the drop is especially sudden in variant 1). This phase occurs after the average technology reaches the frontier technology level (more precisely, the level of technology is close enough to the frontier level, i.e. the firms with technology much worse than the average technology $\langle A\rangle$ have already been eliminated). In this phase, the number of firms stabilizes at the minimum allowed number (with fluctuations resulting from the emergence of spin-offs). The average technology $\langle A\rangle$ grows exponentially in this phase, with growth rate $\sigma$. This phase is stable, i.e. it persists in the $t \rightarrow \infty$ limit.

It is worth to point to the dominating mechanisms of technology growth in the respective phases.

1. Linear technology growth in phase 1 results mainly from the bankruptcies of firms with lowest technology levels.

2. Linear technology growth in phase 2 results from inner technology diffusion, i.e. synergy effects when the firms merge or cooperate to create spinoffs.

3. Exponential technology growth in phase 3 results from outer technology diffusion, i.e. transfer of the frontier technologies. However, since the average level of technology of a given country is in this phase close to the frontier level, the mechanism behind eq. (3) can also be interpreted as own technological improvements of the considered firm.

Now, me move on to the analysis of the sensitivity of the system to the value of the parameter $s$ (sensivity to technological backwardness). This parameter is the main determinant of the dynamics of the system. The dynamics of the average technology $\langle A\rangle$, the average relative technology $\langle A\rangle / F$ and the number of firms $N$ for selected values of the parameter $s$ is depicted in the left of Figs. 6-8 (all plots correspond to variant 1 of the algorithm). 


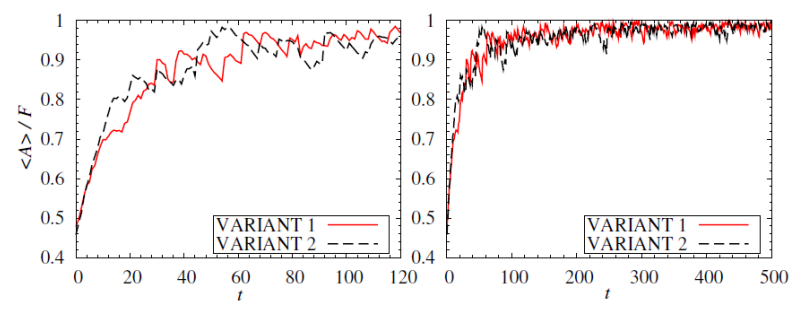

Fig. 4. Average relative technology dynamics in the mid- and long-term.

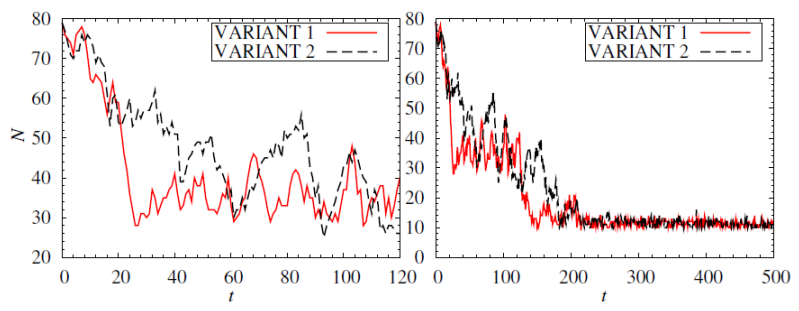

Fig. 5. The dynamics of the number of firms in the mid- and long-term.

In the right, the dependence of the same quantities on the parameter $s$ is shown for fixed time $t=18$.

Very small values of he parameter $s$ mean that there is very little selection pressure on firms with low technology levels (the probability of their bankruptcy is very small). This implies that the growth of technology is very slow and the economy stays for a very long time in the first phase. We also observe that the number of firms can even increase (and reach the maximal allowed number of one firm per site), if the value of $s$ is smaller than ca. $0.1-$ the initially free lattice sites are quickly filled by spin-offs that have a large chance of survival even if their mother firms had very low levels of technology.

Higher values of $s$ mean that the low-technology firms will go bankrupt faster, thus increasing the average level of technology and creating space for other firms to move through the lattice, merge, create spin-offs and in this way profit from inner and outer technology diffusion effects.

While increasing the value of $s$ always leads to a faster

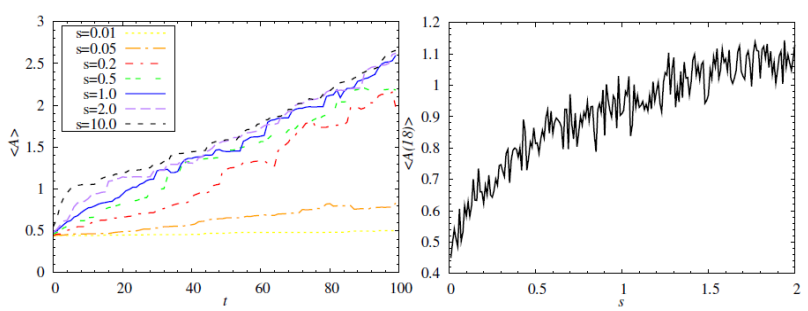

Fig. 6. The dynamics of the average level of technology for selected values of $s$ (left) and the dependence of the average level of technology at $t=18$ on $s$ (right).

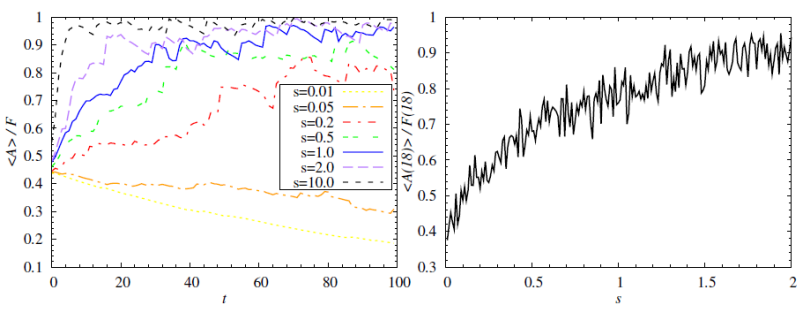

Fig. 7. The dynamics of the average level of relative technology for selected values of $s$ (left) and the dependence of the average level of relative technology at $t=18$ on $s$ (right).

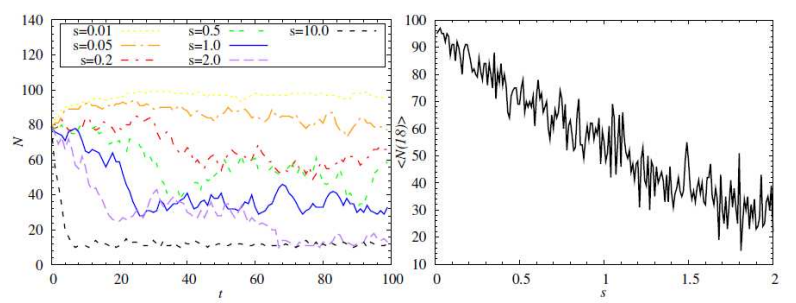

Fig. 8. The dynamics of the number of firms for selected values of $s$ (left) and the dependence of the number of firms at $t=18$ on $s$ (right).

growth at the beginning of the period of analysis, in practical terms values greater than $s \approx 1-1.5$ lead to the same long-term dynamics - the system quickly approaches the third phase (stable equilibrium phase), in which technology grows exponentially with the growth rate of the technological frontier. In the right plots of Figs. 6-7 we then observe that for values of $s$ more than ca. 1.5 the technology level at time $t=18$ does not depend on the value of $s$ and the average level of relative technology is close to 1 .

It is interesting to observe how the transition points between different phases change with changing $s$. For values of $s$ smaller than ca. 0.5100 time steps are not enough to even reach the second phase - the low-technology firms are eliminated very slowly. The transition between the first and second phase is clearly visible for $s=1$, but then 100 time steps are not enough to reach the third phase. The value $s=2$ leads to a faster transition to the second phase (with respect to $s=1$ ) and then around $t=65$ the third phase is approached. If $s=10$, the elimination of low-technology firms is so fast that the second phase is not visible and the system approaches the third phase (with the minimal allowed number of firms) in just around 10 time steps.

\section{Empirical analysis for the OECD countries}

The main parameter that determines the dynamics of the model, in particular the rate of technology growth, is the technological backwardness sensitivity parameter $s$. Therefore, an empirical analysis based on the model can 
consist in determining the values of $s$ for a group of countries and then drawing conclusions about the underlying mechanisms of technology growth in these countries.

The analysis was performed for the years 1981-1999 and for 28 countries which were Organisation for Economic Co-operation and Development (OECD) members in the period of analysis (some of them joined in the middle of this period).

The economy of each country was modelled as a $10 \times 10$ system with an initial concentration $c_{0}=0.8$. The minimal number of firms in each country was set to $N_{\min }=10$ and the parameter $b$ was chosen to be 0.01 .

The initial (1981) technology level for the $i$-th country was assumed to be at its empirical level $A_{i}^{e m p}(0)$ (relatively to USA, which was assumed to be the technological leader with $\left.A_{\mathrm{USA}}^{e m p}(0)=1\right)$. To obtain the actual (empirical) distribution of technology levels, the economic agents in the modelled systems were assigned technology levels $A$ from the uniform distribution on the interval $\left(0,2 A_{i}^{e m p}(0)\right)$, if $A_{i}^{e m p}(0) \leq 0.5$, or on the interval $\left(1-2 A_{i}^{e m p}(0), 1\right)$, if $A_{i}^{e m p}(0)>0.5$ (thus, no agent had an initial technology of more than 1 and USA were really the technological leader).

The technology level for USA (the frontier technology) was assumed to grow at the average empirical USA growth rate of $2.08 \%$ (in the period 1981-1999). Hence, the technology frontier function for all other countries was given by $F(t)=e^{0.0208 t}$. To see the empirical dynamics of USA technology levels and the prediction of this simple exponential function, see Fig. 9. The criterion to choose the frontier growth rate was chosen in such a way that $F(18)$ matched the empirical USA technology level in 1999.

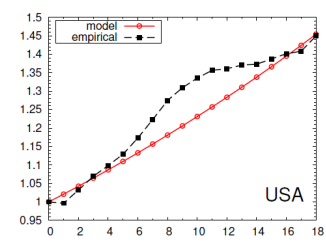

Fig. 9. Empirical technology dynamics for USA and the assumed model dependence for the frontier technology $F(t)=e^{0.0208 t}$.

We now define the mean relative error (MRE) that measures how well our model with a given value of the parameter $s$ describes empirical dynamics for the analyzed country, indexed by $i$ :

$$
M R E_{i}=\sum_{t=0}^{18} \frac{\left|A_{i}(t)-A_{i}^{e m p}(t)\right|}{A_{i}^{e m p}(t)},
$$

where $A_{i}(t)$ denotes the level of technology that results from the model at time $t$ and $A_{i}^{e m p}(t)$ the empirical level of technology at time $t$.

The value of the parameter $s$ that minimizes MRE for a given country was then chosen as the calibrated value that pertains to this country.

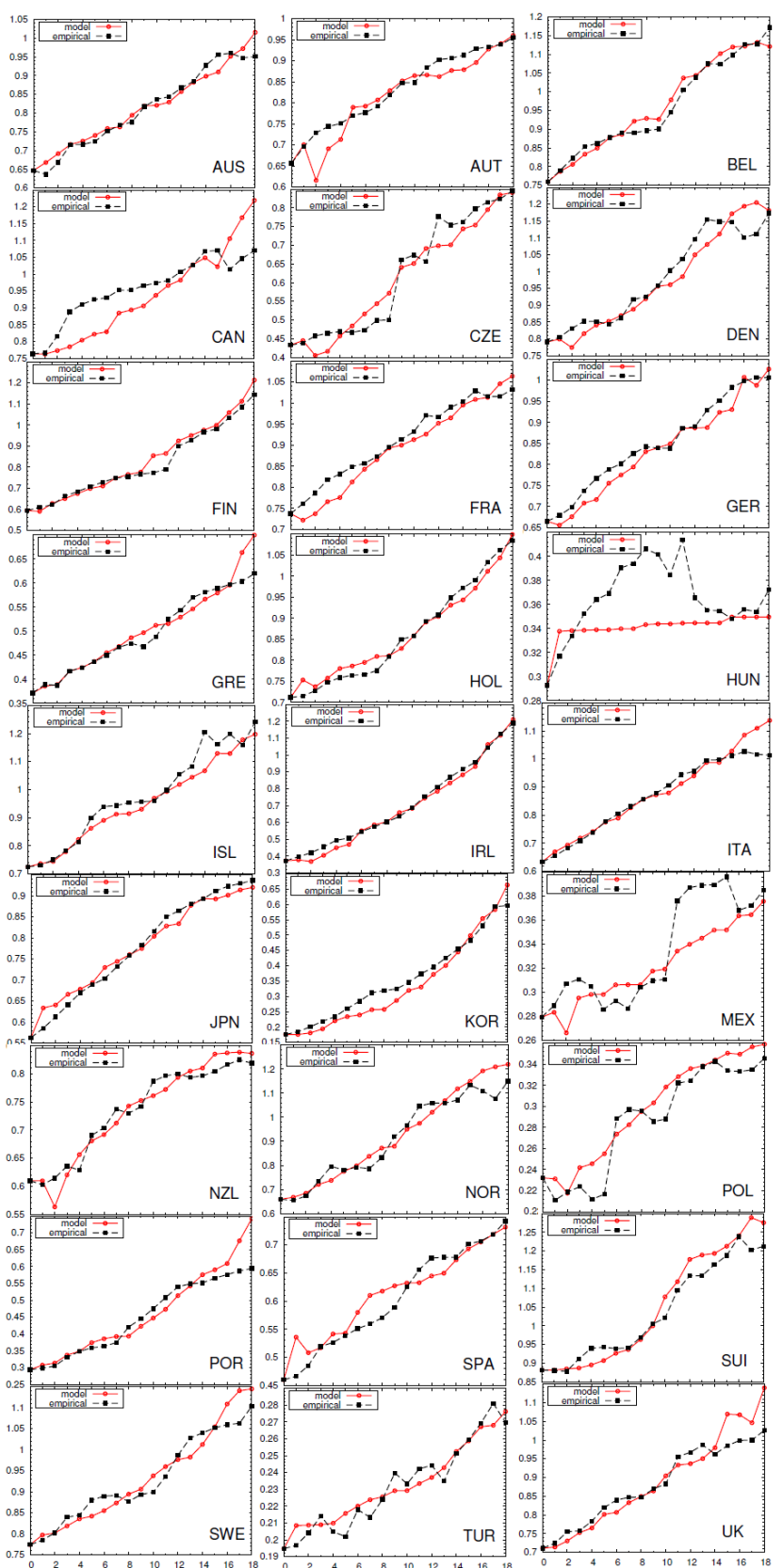

Fig. 10. Empirical and model technology dynamics for 27 OECD countries.

The empirical and model technology dynamics for all countries are shown in Fig. 10 and the calibrated values of the parameter $s$ (together with MREs of calibration) are shown in Tab. I. An important property of the model curves is the presence of random irregularities. These irregularities are indeed random and since their magnitude is very similar to the magnitude of empirical fluctuations, we can suppose that the empirical fluctuations are also random - in the sense that they do not reflect any particular mechanism (e.g. some cyclical behaviour).

In general, the agreement between the model and empirical data is rather good for most of the countries and 
a large part of MREs (which varies from ca. $1.9 \%$ to ca. $10 \%$ ) for each country is due to the random fluctuations in model and empirical curves.

TABLE I

The results of the calibration of the parameter $s$ and the corresponding mean relative errors (in \%)

\begin{tabular}{ccc|ccc|ccc}
\hline \hline C'try & $s$ & $M R E$ & C'try & $s$ & $M R E$ & C'try & $s$ & $M R E$ \\
\hline AUS & 0.80 & 2.17 & GRE & 0.58 & 2.77 & NZL & 0.46 & 2.63 \\
AUT & 0.70 & 3.39 & HUN & 0.02 & 8.21 & NOR & 1.73 & 4.05 \\
BEL & 1.46 & 1.86 & IRL & 1.38 & 4.22 & POL & 0.73 & 5.37 \\
CAN & 1.87 & 6.86 & ISL & 1.29 & 3.29 & POR & 0.74 & 5.61 \\
CZE & 0.55 & 5.77 & ITA & 1.66 & 2.53 & SPA & 0.32 & 3.69 \\
DEN & 1.34 & 3.46 & JPN & 0.48 & 2.25 & SWE & 1.15 & 2.73 \\
FIN & 0.97 & 2.81 & KOR & 1.38 & 9.95 & SWI & 1.98 & 2.65 \\
FRA & 1.69 & 3.04 & MEX & 0.39 & 6.32 & TUR & 0.36 & 2.82 \\
GER & 0.94 & 2.84 & NED & 0.83 & 2.04 & UK & 1.36 & 3.28 \\
\hline
\end{tabular}

We now want to draw some conclusions from the calibrated values of the parameter $s$. In particular, we would like to see whether similar countries (e.g. developing ones) have similar values of this parameter. In Tab. I, one can see that the obtained values of $s$ are very different for different countries and vary from 0.02 (Hungary this country, however, has very strange empirical dynamics and thus we can not conclude much about it) to 1.98 (Switzerland). Therefore, taking into account our analysis of the properties of this model, we can distinguish three groups of countries:

- countries in which the inner technology diffusion dominates (low values of $s$ ),

- countries in which the inner and outer technology diffusion play a similar role $(s \approx 1)$,

- countries in which the outer technology diffusion or autonomous innovations dominate (high values of $s)$.

The introduced classification of countries is of course not very strict. To classify the countries, we assume that low value of $s$ means less than $0.8, s \approx 1$ means $s \in[0.8,1.2]$ and $s>1.2$ means a high value of $s$.

The first group of countries consists of: Austria, the Czech Republic, Greece, Hungary, Japan, Mexico, New Zealand, Poland, Portugal, Spain and Turkey. These are mostly developing countries, in which the technological advancement is not the most important factor that determines the probability of firm survival (hence, $s$ is low). The presence of Japan means that low sensitivity to technological backwardness can also be the property of the most developed countries. This is also plausible, since Japan belongs to the strict group of world technological leaders and the firms with low technology levels have gone bankrupt a long time ago - hence now the value of $s$ is not essential any more.
The third group of countries consists almost entirely of highly-developed countries (Belgium, Canada, Denmark, France, Ireland, Iceland, Italy, Norway, South Korea, Switzerland, United Kingdom). The only exception is South Korea, which was a developing country in the period of interest). In this group of countries, which have long traditions of free market economy, the level of technology is one of the most important factors of competition between companies and the underdeveloped firms do not survive for long.

The second group of countries consists of a relatively small number of countries (Austria, Finland, Germany, Holland, Sweden) and it can be interpreted as interpolating between the two other groups. E.g. Germany was divided into two independent states for half of the analyzed period and hence it shows the properties of both groups (developing and highly-developed countries).

The general conclusion from this empirical analysis is that the sensitivity to technological backwardness is rather small in the developing countries and rather high in the highly-developed states. We may therefore suppose that the mechanisms of technological progress are different in the developing and highly-developed countries. In the former, technological progress stems mainly from technology transfers from the latter (e.g. from international firms that invest in the developing countries). In the latter, in turn, the levels of technology of firms are not only higher, but also less diversified. Hence, the dominating mechanism of technological progress is the development of firms' own innovations or the use of the world most developed technologies.

\section{Conclusion}

In this paper we have investigated a modification and reinterpretation of a model introduced by Ausloos et al. The model describes a set of economic agents who can move through a lattice, merge, create spin-offs etc. and thus increase their technology levels. We have analyzed the properties of the model and we have distinguished three phases in its dynamics. The main parameter that governs the dynamics of the model is the sensitivity to technological backwardness. We have also performed an empirical analysis of the model for a group of 28 OECD countries. We have divided the countries into 3 groups and we have speculated about the mechanisms of technological progress in them.

Econophysical analysis of technological progress is rather new. However, it seems to be interesting and it can give new perspective on one of the most important determinants of economic growth. The advantage of an econophysical way of modelling is that any sensible set of agents behaviour rules can easily be implemented in a Monte Carlo-type simulation. In this paper we have shown that it is possible to perform empirical research based on such model. However, of course if such models are to become a realistic description of actual economies, a lot of work needs to be done. 


\section{Acknowledgments}

The author thanks Prof. Krzysztof Malaga and members of Chair of Mathematical Economics of the Poznań University of Economics for interesting discussions. I have also profited from a discussion with Dr. Pawel Sobkowicz during FENS 2010 who suggested to me interesting ways of improving the model.

\section{References}

[1] A. Smith, An Inquiry into the Nature and Causes of the Wealth of Nations, W. Strahan and T. Cadell, London 1776.

[2] F. Ramsey, Journal of Political Economy 38, 543 (1928).

[3] R. Solow, Quarterly Journal of Economics 70, 65 (1956).

[4] T. Swan, Economic Record 32, 334 (1956).

[5] D. Cass, Review of Economic Studies 32, 233 (1965).

[6] T. Koopmans, in: The Econometric Approach to Development Planning, North Holland, Amsterdam 1965, p. 225.

[7] P. Romer, Journal of Political Economy 98, S71 (1990).
[8] R. Lucas, Journal of Monetary Economics 22, 3 (1988).

[9] K. Cichy, Human capital and technological progress as the determinats of economic growth, Working Paper No. 60, National Bank of Poland, pp. 1-156, Warsaw 2009 .

[10] B. Chakrabarti, A. Chakraborti, A. Chatterjee (eds.), Econophysics and Sociophysics, Wiley-VCH Verlag, Weinheim 2011.

[11] M. Gallegati, A.P. Kirman, M. Marsili (eds.), The Complex Dynamics of Economic Interaction, Springer-Verlag, Berlin, Heidelberg 2004.

[12] M. Ausloos, P. Clippe, A. Pekalski, Physica A 337, 269 (2004), arXiv:cond-mat/0401144.

[13] D. Delli Gatti, C. Di Guilmi, E. Gaffeo, G. Giulioni, M. Gallegati, A. Palestrini, arxiv: cond-mat/0312096 (2003).

[14] M. Llas, P. Gleiser, J. Lopez, A. Diaz-Guilera, Physical Review E 68, 66101 (2003), arXiv:condmat/0309544.

[15] M. Szydlowski, A. Krawiec, Acta Physica Polonica B 37, 3161 (2006). 